Chapter 9

\title{
Graphene Nanowalls
}

\author{
Mineo Hiramatsu, Hiroki Kondo and Masaru Hori
}

Additional information is available at the end of the chapter

http://dx.doi.org/10.5772/51528

\section{Introduction}

Graphene, hexagonal arrangement of carbon atoms forming one-atom thick planar sheet, is a promising material for future electronic applications due to their high electrical conductivity as well as chemical and physical stability [1]. Planar graphene films with respect to the substrate have been synthesized using various methods including mechanical exfoliation from highly oriented pyrolytic graphite, chemical exfoliation from bulk graphite, thermal decomposition of carbon-terminated silicon carbide, and chemical vapor deposition (CVD) on metals such as nickel and copper substrates [2-6].

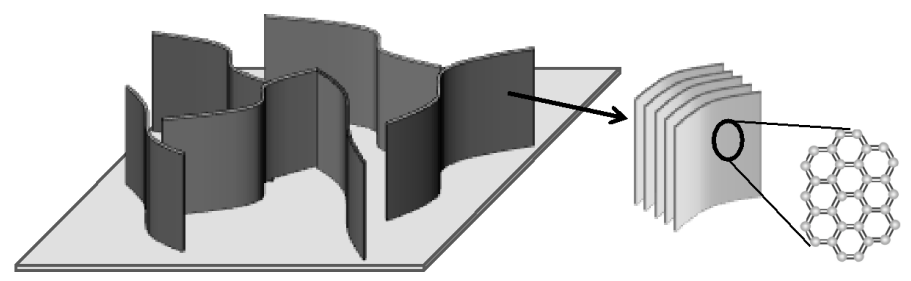

Figure 1. Schematic illustration of graphene nanowalls

On the other hand, plasma-enhanced CVD (PECVD) is among the early methods to synthesize of vertically standing few layer graphenes or graphene nanowalls (GNWs) [7-12]. GNWs can be described as self-assembled, vertically standing, few-layered graphene sheet nanostructures, which are also called as carbon nanowalls, carbon nanosheets, and carbon nanoflakes. As illustrated in Fig. 1, the sheets form a self-supported network of wall structures with thicknesses ranging from a few nanometers to a few tens of nanometers. GNWs have a high density of atomic scale graphitic edges that are potential sites for electron field emission, which might lead to the application in flat panel displays and light sources $[13,14]$. 
The large surface area of GNWs is useful as templates for the fabrication of other types of nanostructured materials, electrodes for energy storage devices and biosensors [15-20]. These graphene-based materials are applied in the field of electrochemistry including electrode for fuel cell and electrochemical sensors. In these applications, graphene-based materials are often decorated with metal nanoparticles and other materials.

In this chapter, basic properties of GNWs and their prospective applications are described. First of all, synthesis and characterization of GNWs are outlined. PECVD is becoming one of the most promising techniques for the production of carbon materials including diamond, aligned carbon nanotube films and GNWs, due to its feasibility and potentiality for largearea production with reasonable growth rates at relatively low temperatures. In the present study, GNW growth using inductively coupled plasma (ICP) enhanced CVD is featured, since the ICP CVD system has advantages of simple design and scalability to large area growth. The growth mechanism, characterization of GNWs, and several decoration techniques of GNW surface are described.

Due to the large surface area of GNWs, we can expect a variety of electrochemical applications using GNWs such as batteries, capacitors, and sensors. To these ends, GNWs are often decorated with nanoparticles or films. In the latter half of this chapter, application of GNWs as electrode for fuel cell is described. GNWs were grown on the carbon fiber paper. Then metal organic chemical fluid deposition (MOCFD) using supercritical fluid (SCF) was applied to form platinum $(\mathrm{Pt})$ nanoparticles on the surface of GNWs. Using this method, highly dispersed Pt nanoparticles of approximately $2 \mathrm{~nm}$ in diameter were formed on the surface of GNWs grown on the carbon fiber paper. Furthermore, the application as a biosensor using GNWs is described. As another example, the electrocatalytic activity of GNWs for determining dopamine, ascorbic acid and uric acid in phosphate buffer solution was investigated. The ability of GNWs as a platform to create graphene-based hybrid materials is demonstrated.

\section{Growth of graphene nanowalls and their growth mechanism}

\subsection{Growth of graphene nanowalls using inductively coupled plasma CVD}

Synthesis techniques for GNWs and related vertical graphene structures are similar to those used for diamond films and carbon nanotubes (CNTs). In general, a mixture of hydrocarbon and hydrogen or argon gases, typically $\mathrm{CH}_{4}$ and $\mathrm{H}_{2}$, is used as source gases for the synthesis of GNWs. Unlike the CNT growth, GNWs can be fabricated on a variety of substrates, including $\mathrm{Si}, \mathrm{SiO}_{2}, \mathrm{Al}_{2} \mathrm{O}_{3}, \mathrm{Ni}, \mathrm{Ti}$, and stainless steel, at substrate temperatures of $500-750{ }^{\circ} \mathrm{C}$ without the use of catalysts [8]. To date, GNWs have been grown using various PECVD methods employing microwave plasma, inductively coupled plasma (ICP), capacitively coupled plasma (CCP) with $\mathrm{H}$ radical injection, very high frequency (VHF) plasma with $\mathrm{H}$ radical injection, electron beam excited plasma, and DC plasma [7-12,19].

Radio frequency (RF: $13.56 \mathrm{MHz}$ ) ICP is one of high-density plasmas, and has been used to etch several materials including $\mathrm{Si}, \mathrm{SiO}_{\mathrm{x}}, \mathrm{SiN}_{\mathrm{x}}$, and metal films in the LSI fabrication process. The ICP is operated at relatively low pressures below $100 \mathrm{mTorr}$ (13.3 Pa). In the case of pla- 
nar geometry, RF power is inductively coupled into the process chamber with a planar-coil antenna through a quartz (fused silica) window, and plasma is generated in the chamber. Plasma density of ICP discharge is on the order of $10^{12} \mathrm{~cm}^{-3}$. Figure 2 shows a schematic of ICP reactor with planar geometry used for the growth of GNWs. The ICP reactor was $16 \mathrm{~cm}$ in diameter and $30 \mathrm{~cm}$ in height. A one-turn coil antenna with a diameter of $10 \mathrm{~cm}$ was set on a quartz window at the top of reactor. $\mathrm{Si}$ or $\mathrm{SiO}_{2}$-coated $\mathrm{Si}$ substrates were set on the middle of the substrate holder at $10 \mathrm{~cm}$ below the quartz window.

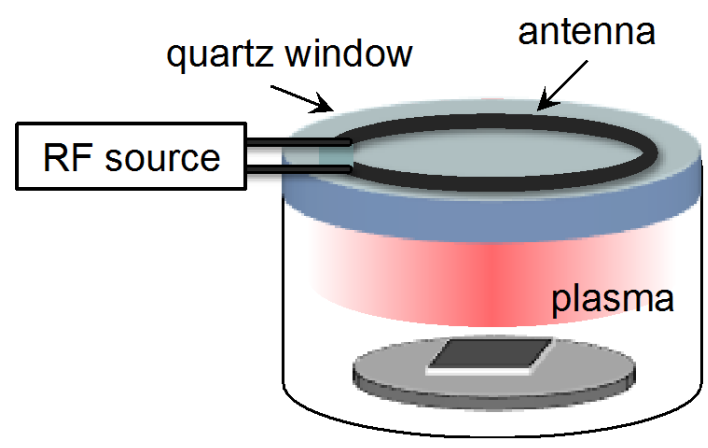

Figure 2. Schematic of inductively coupled plasma reactor with planar geometry used for the growth of GNWs.
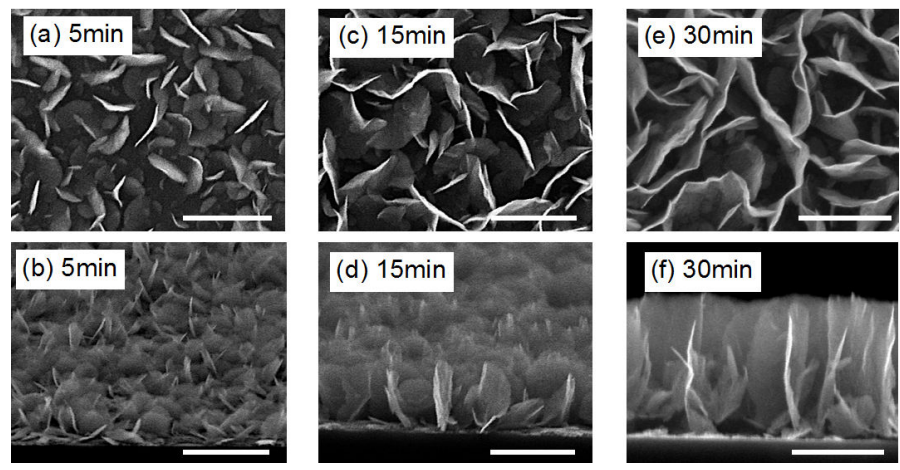

Figure 3. SEM images of the GNW films grown by ICP-CVD employing a mixture of $\mathrm{CH}_{4}$ and $\operatorname{Ar}$ for (a-b) 5 min, (c-d) 15 min, and (e-f) $30 \mathrm{~min}$. Scale bar: $1 \mu \mathrm{m}[21]$.

Figures 3(a) -3(f) show scanning electron microscopy (SEM) images of GNW films grown by ICP-CVD employing a mixture of $\mathrm{CH}_{4}$ and Ar for different growth periods. After the nucleation stage of GNWs, growth of less-aligned, isolated carbon sheets with a semicircular shape standing on the substrate is confirmed as shown in Figs. 3(a) and 3(b). As the growth period increased, density of isolated nanosheets increased and those standing almost vertically on the substrate continued preferably to spread faster. Then, spreading nanosheets met one an- 
other; eventually resulting in the formation of linked nanowalls as shown in Figs. 3(c) and $3(d)$. During the early growth stage after the nucleation up to the steady-state growth, the growth for the inclined smaller nanowalls was terminated, while the vertical nanowalls preferentially continued to grow. Therefore, with the increase of growth period in the early stage, the spacing between nanowalls at their top increased gradually, and then became almost saturated, resulting in the formation of two-dimensional carbon sheets standing vertically on the substrate with high aspect ratio. As shown in Figs. 3(e) and 3(f), with the further increase of growth period during the steady-state growth, the height of vertical aligned GNWs increased, while the thickness of nanowalls and the spacing between nanowalls became almost saturated with keeping the morphology of GNWs.

Growth rate curve for the GNWs fabricated by ICP-CVD employing $\mathrm{CH}_{4} / \mathrm{Ar}$ system was obtained by measuring the height of the nanowalls for differing period of growth (0-120 min). Figure 4 shows the average height of GNW films as a function of growth period. As shown in Fig. 4, the height of GNWs almost linearly increased with the increase of growth period in the range from 10 to $120 \mathrm{~min}$, while it took approximately $5 \mathrm{~min}$ for the nucleation. The growth rate of GNWs in the steady-state condition was constant at approximately $60 \mathrm{~nm} / \mathrm{min}$.

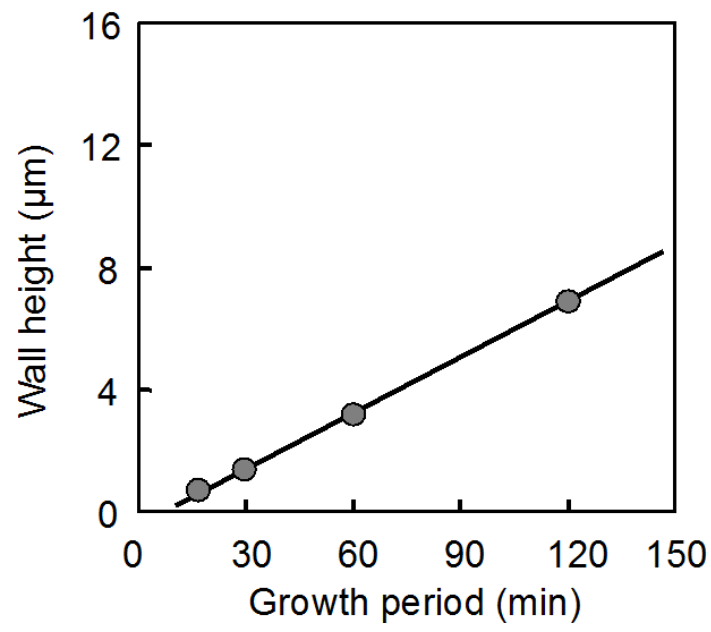

Figure 4. Wall height of GNWs as a function of growth period. The growth rate data were obtained from the samples grown for different period on Si substrates by ICP-CVD employing $\mathrm{CH}_{4} / \mathrm{Ar}$ system [21].

\subsection{Characterization of graphene nanowalls}

As was illustrated in Fig. 1, GNWs can be described as graphite sheet nanostructures with edges that are composed of stacks of graphene sheets standing almost vertically on the substrate. The sheets form a self-supported network of wall structures with thicknesses in the range from a few nanometers to a few tens of nanometers, and with a high aspect ratio. In this section, typical GNWs grown using PECVD are characterized using SEM, transmission 
electron microscopy (TEM), in-plane synchrotron X-ray diffraction, and Raman spectroscopy using a $514.5 \mathrm{~nm}$ line of argon laser.

Figure 5(a) shows a typical SEM image of GNW film, indicating the vertical growth of the two-dimensional carbon sheets with honeycomb structure on the substrate. Actually, the morphology and structure of GNW film depend on the source gases, pressure, process temperature, as well as the type of plasma used for the growth of GNWs. In addition to the vertically standing maze-like structure, isolated very thin nanosheets, less aligned petal-like, highly branched type, and a kind of porous film have been fabricated so far.

Figure 5(b) shows a low-magnification TEM image of a piece of typical GNW of a micrometer-high planar nanosheet structure with a relatively smooth surface. The GNW is composed of nano-domains of a few tens of nanometers in size. The high-resolution TEM image of the GNW shown in Fig. 5(c) reveals the graphene layers, which indicates the graphitized structure of the GNWs. The spacing between neighboring graphene layers was measured as approximately $0.34 \mathrm{~nm}$.
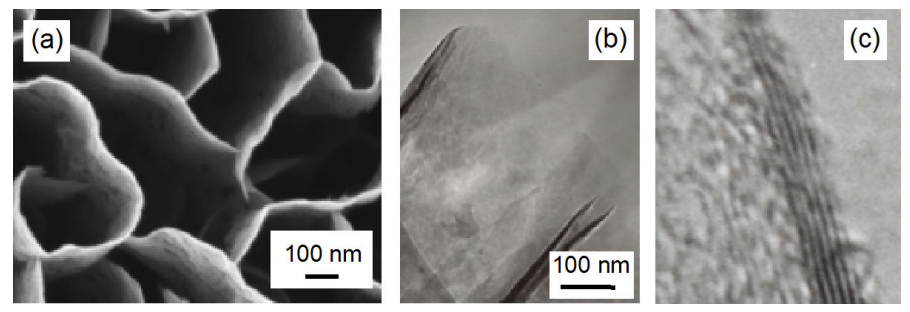

Figure 5. a) Typical SEM image of GNW film, (b) low-resolution TEM image of GNW on a microgrid, and (c) high-resolution TEM image of GNW, showing graphene layers at the fold of GNWs.

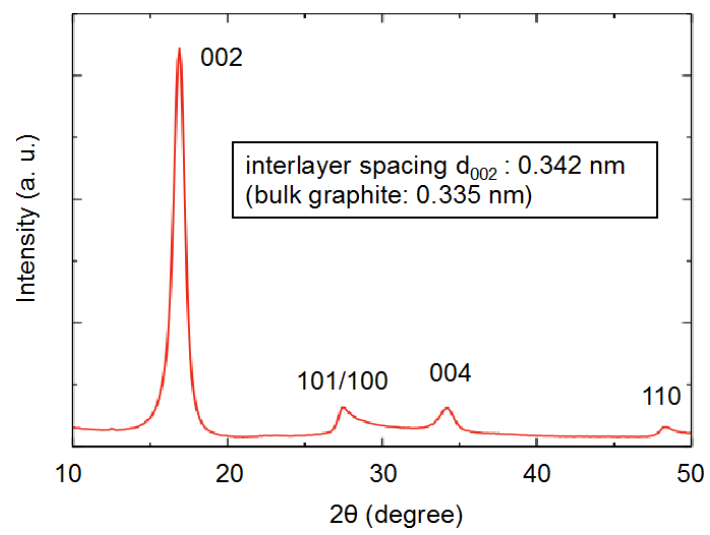

Figure 6. SR X-ray diffraction pattern of GNW film measured at beam line BL13XU of SPring-8 [22]. 
The crystallinity of GNWs was analyzed using synchrotron X-ray surface diffraction at grazing incidence and exit at the beamline BL13XU of SPring-8 [22]. The in-plane diffraction technique measures diffracted beams, which are scattered nearly parallel to the sample surface and hence measures lattice planes that are perpendicular to the sample surface. The Xray beam was incident on the GNW film sample at grazing angle of $0.3^{\circ}$ relative to the substrate surface. Figure 6 shows the grazing incidence in-plane X-ray diffraction pattern of GNW film sample. An intense 002 Bragg peak, the plane of which is normal to the substrate, is at $2 \theta=16.9^{\circ}$ and there are also weak $100 / 101,004$, and 110 Bragg peaks. The interlayer spacing $\mathrm{d}_{002}$ was determined from the 002 peak by applying Bragg's law with a wavelength of $0.1003 \mathrm{~nm}$. It was found to be $0.342 \mathrm{~nm}$ for all samples, which is slightly larger than that of bulk graphite $(0.335 \mathrm{~nm})$.

Raman spectrum for GNWs grown on Si substrate is shown in Fig. 7. Typical Raman spectrum for the GNWs has G band peak at $1590 \mathrm{~cm}^{-1}$ indicating the formation of a graphitized structure and $\mathrm{D}$ band peak at $1350 \mathrm{~cm}^{-1}$ corresponding to the disorder-induced phonon mode. The peak intensity of $\mathrm{D}$ band is twice as high as that of $\mathrm{G}$ band. The $\mathrm{G}$ band peak is accompanied by a shoulder peak at $1620 \mathrm{~cm}^{-1}$ (D' band), which is associated with finite-size graphite crystals and graphene edges [23,24]. The 2D band peak at $2690 \mathrm{~cm}^{-1}$ is used to confirm the presence of graphene and it originates from a double resonance process that links phonons to the electronic band structure $[25,26]$. The strong and sharp D band peak and D' band peak suggest a more nanocrystalline structure and the presence of graphene edges and defects, which are prevalent features of GNWs [8,10,12].

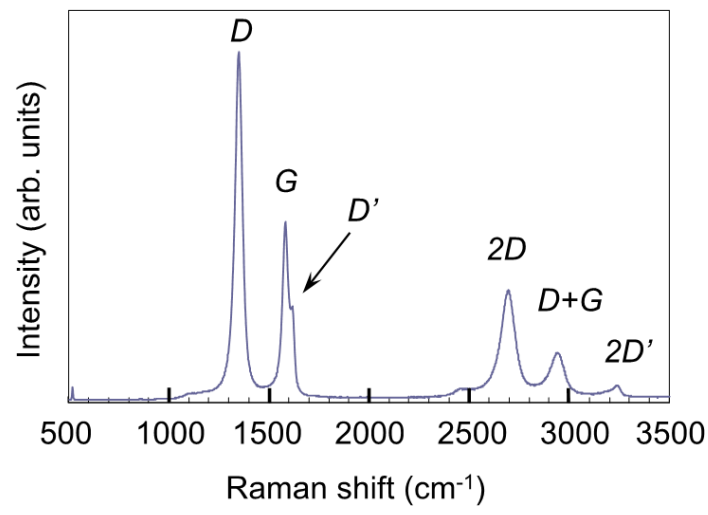

Figure 7. Typical Raman spectrum of GNWs measured using the $514.5 \mathrm{~nm}$ line of an Ar laser [21]

\subsection{Nucleation of vertical nanographenes}

From the temporal dependence of nanowall height shown in Fig. 3, nucleation of GNWs is considered to occur before the commencement of steady-state growth. Significant interest exists in clarifying the nucleation mechanism of GNWs at the very early stage. Figure 8(a) shows a top view $\mathrm{SEM}$ image of $\mathrm{SiO}_{2}$ substrate surface after 30 sec growth. First the surface of substrate was 
covered with amorphous carbon layer, which is later confirmed using Raman spectroscopy. In $1 \mathrm{~min}$, the onset of nano-graphene growth was observed in the top view SEM image of deposits formed for $1 \mathrm{~min}$ as shown Fig. 8(b). Figures 8(c) and 8(d) show tilted SEM image and crosssectional TEM image of deposits, respectively, formed for $2 \mathrm{~min}$. For the cross-sectional TEM observation, sample surface was coated with the epoxy resin, the deposits embedded in the epoxy resin were peeled off from the substrate, and then the substrate side (interface side) of resin embedding the deposits was coated again with the epoxy resin. In Fig. 8(d), the red line corresponds to the interface to the substrate surface and the red arrow indicates the growth direction. In $2 \mathrm{~min}$, the formation of isolated graphene sheets was observed on the amorphous carbon layer as shown in Fig. 8(c). The thickness of amorphous carbon layer was estimated to be $30 \mathrm{~nm}$ from the TEM observation shown in Fig. 8(d).
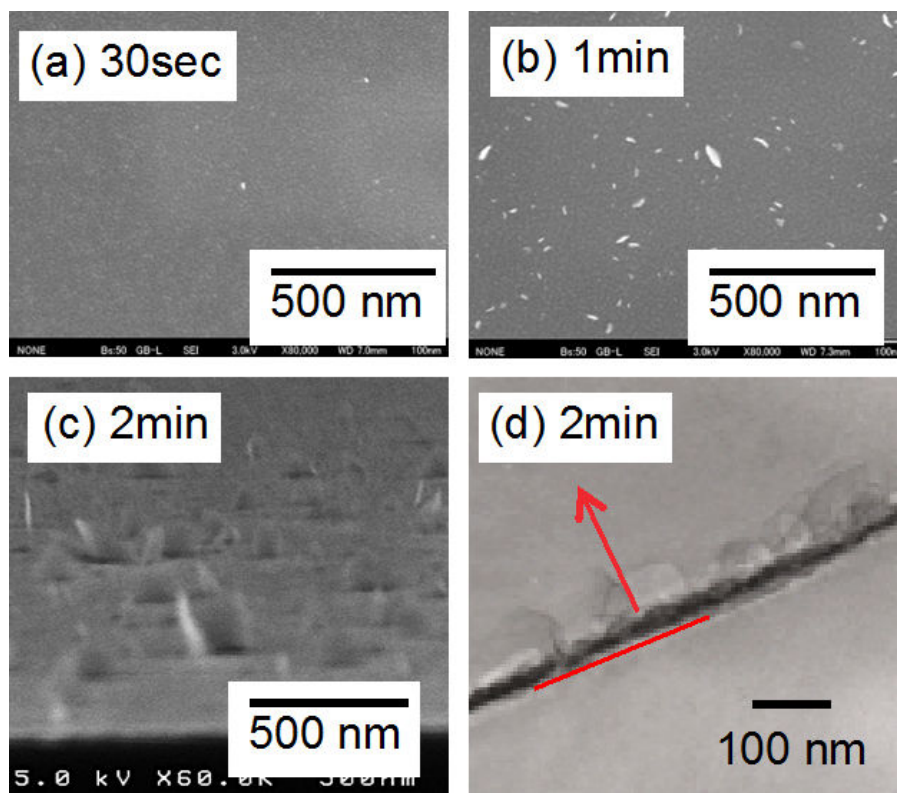

Figure 8. SEM and TEM images of deposits formed at the nucleation stage. (a) Top view SEM image of substrate surface after $30 \mathrm{sec}$. (b) Top view SEM image of deposits formed for $1 \mathrm{~min}$, indicating the commencement of nano-graphene growth. (c) Tilted SEM image of deposits formed for 2 min. (d) Cross-sectional TEM image of deposits formed for $2 \mathrm{~min}$. Deposits were detached from the Si substrate. Red line in (d) corresponds to the interface to the substrate surface and the red arrow indicates the growth direction [21].

Figure 9 shows Raman spectra of deposits formed on $\mathrm{SiO}_{2}$-coated $\mathrm{Si}$ substrate in the nucleation stage. The peak around $950 \mathrm{~cm}^{-1}$ originates from the Si wafer [27]. The broad peak at $1340 \mathrm{~cm}^{-1}$ in the Raman spectrum at $30 \mathrm{sec}$ indicates that the deposits are amorphous carbon or diamond-like carbon. Namely, during the nucleation period, graphene component was scarcely contained in the underlying interface layer shown in Fig. 9. 
So far, several papers have been published on the observation of GNW growth in the early growth stage and the nucleation mechanism for the formation of vertical layered-graphenes using various CVD methods [8,28-35]. The things in common in previous observations are that there is an induction period of $1-5 \mathrm{~min}$ before the onset of vertical nano-graphene growth and an interface layer exists between vertical nano-graphenes and the surface of $\mathrm{Si}$ and $\mathrm{SiO}_{2}$ substrates. $\mathrm{Zhu}$, et al. [31,32] reported the presence of graphenes parallel to the substrate surface before the onset of vertical nanosheet growth, although neither Raman spectrum nor TEM image of base layer was attached. In their model, these few-layer graphenes would grow parallel to the substrate surface until a sufficient level of force develops at the grain boundaries to curl the leading edge of the top layers upward. The vertical orientation of these sheets would result from the interaction of the plasma electric field with their anisotropic polarizability [32]. In the case of GNW growth in the present work, on the other hand, the interface layer under the CNWs was an amorphous or diamond-like carbon, which is similar to the cases using radical injection PECVD, multi-beam CVD, and DC PECVD $[8,28-30,33,34]$. Moreover, ion bombardment on the surface will play an important role for nucleation by creating active sites for neutral radical bonding [30]. The existence of amorphous or diamond-like carbon interface layer will enable us to grow GNWs and similar structures on a variety of substrates without catalyst.

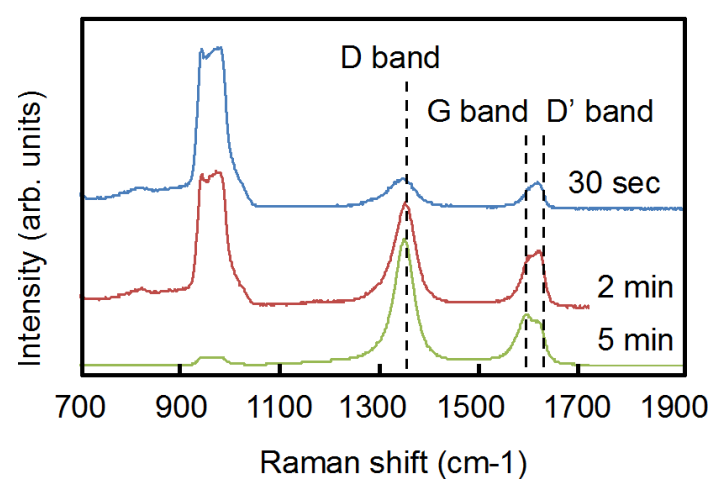

Figure 9. Raman spectra of deposits formed in the nucleation stage on $\mathrm{SiO}_{2}$-coated Si substrate. Peak around $950 \mathrm{~cm}^{-1}$ originates from the Si wafer [8].

\subsection{Growth mechanism of graphene nanowalls}

Figure 10 depicts the initial growth process of GNWs. A model for the initial growth mechanism is as follows. (1) In the beginning, hydrocarbon radicals such as $\mathrm{CH}_{3}$ are adsorbed on the substrate, forming a very thin amorphous carbon layer. Ion irradiation induces the formation of dangling bonds on the growing surface, resulting in the formation of nucleation sites. (2) Adsorbed carbon species are migrating on the surface and condensed to form nanoislands with dangling bonds. (3) Ion irradiation would also enhance the adsorption of $\mathrm{CH}_{x}$ radicals on the surface. (4) Small, disordered graphene nanosheets are nucleated at these 
dangling bonds, followed by two-dimensional growth and subsequent formation of nanographene sheets with random orientation. (5) Among the nucleated graphene sheets with random orientations, those standing almost vertically on the substrate continued preferably to grow up faster to vertically standing nanosheets owing to the difference in the growth rates along the strongly bonded planes of graphene sheets expanding and in the weakly bonded stacking direction. Reactive carbon species arriving at the edge of the graphene layer are easily bonded to the edge, and eventually the graphene layer would expand preferably along the direction of radical diffusion, perpendicular to the electrode plane. On the other hand, low-lying inclined graphene sheets were shadowed by the high-grown vertical graphene sheets. As a result, the amounts of reactive carbon species arriving at the low-lying inclined graphene sheets decreased, resulting in the termination of growth for the inclined smaller nanowalls, while the vertical nanowalls preferentially continued to grow. As growth period increased, spreading vertical nanowalls met one another, eventually resulting in the formation of linked nanowalls similar to a maze. With further increase of growth period, the spacing between nanowalls at their top increased gradually, and then became almost saturated, resulting in the formation of two-dimensional graphene sheets standing vertically on the substrate with high aspect ratio. In the steady-state growth condition, the height of nanowalls increased almost linearly with keeping their morphology.

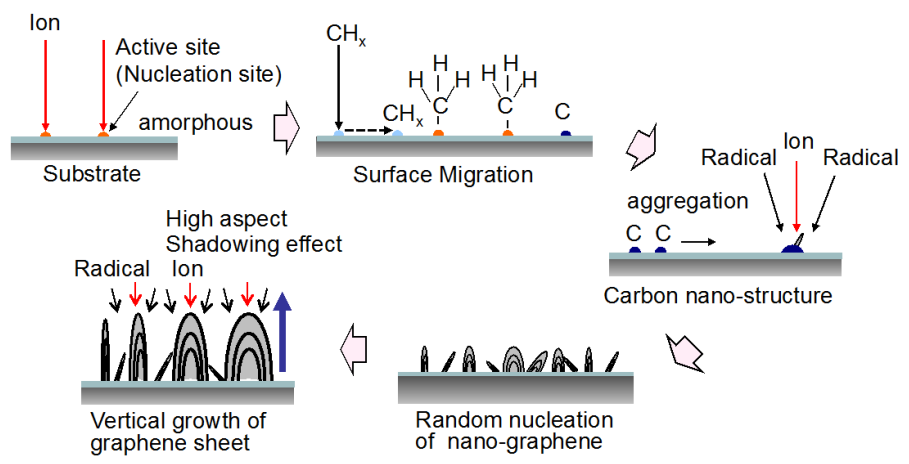

Figure 10. Illustration of the initial growth model of GNWs

\section{Functionalization of graphene nanowalls}

\subsection{Decoration of graphene nanowall surface}

Due to the large surface area (high surface-to-volume ratio) of GNWs, we can expect a variety of electrochemical applications using GNWs such as batteries, capacitors, and gas sensors. To these ends, GNWs are decorated with nanoparticles or films of metals, semiconductors, and insulators, by using several techniques including vacuum evaporation, sputtering, CVD, and plating. Figure 11 shows schematic illustrations of decorated 
GNWs with different morphologies. GNWs are used as the templates to fabricate other types of nanostructures. The morphology of GNWs decorated with nanoparticles or film depends on the deposition methods of materials. Conformal deposition (Fig. 11(a)) and gap filling (Fig. 11(b)) will be achieved using metal-organic chemical vapor deposition (MOCVD), sputtering, atomic layer deposition, and plating in liquid phase. In Fig. 11(c), thin film or aggregation of nanoparticles would be formed on the top edges of GNWs. Diamond surface is modified with several types of surface termination, e.g. C-NH, $\mathrm{C}-\mathrm{OH}$, and C$\mathrm{COOH}$, and DNA and proteins were immobilized on the surface of diamond and nanodiamond films for bio-sensing application [35-38]. As is the case with the diamond surface, the edges of GNWs can also be modified with similar surface termination, and covalent immobilization of DNA and proteins on the GNWs will be realized. Previously, Wu et al. used GNWs as templates to fabricate large surface area materials, including conformal deposition of $\mathrm{Au}$ and $\mathrm{Cu}$ by electron beam evaporation; conformal deposition of $\mathrm{ZnO}, \mathrm{TiO}_{2}$, $\mathrm{SiO}_{x}, \mathrm{SiN}_{x}$, and $\mathrm{AlO}_{x}$ by atomic layer deposition; conformal deposition of $\mathrm{Ni}$, NiFe and CoNiFe nanoparticles by electrochemical deposition; gap filling with dispersed Fe nanoparticles by the immersion of GNWs into a mixed solution of Fe particles and isopropanol in an ultrasonic bath; Se deposition on the top of edges of GNWs by electrochemical deposition [39-41]. In Fig. 11(d), on the other hand, metal nanoparticles are dispersed on the surface of GNWs, which is a kind of nanocomposite. This morphology can be moderately achieved by plating, sputtering, and laser ablation.

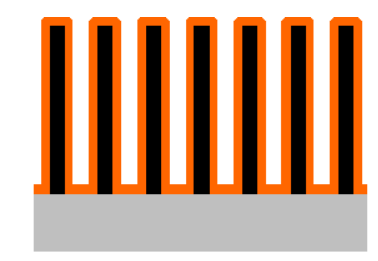

(a)

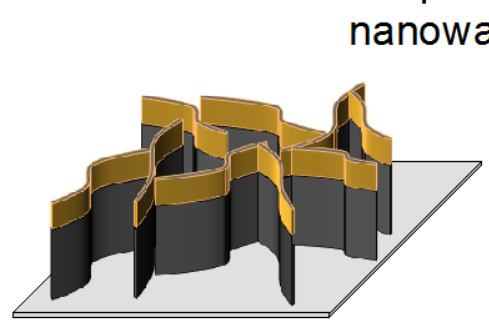

(c)

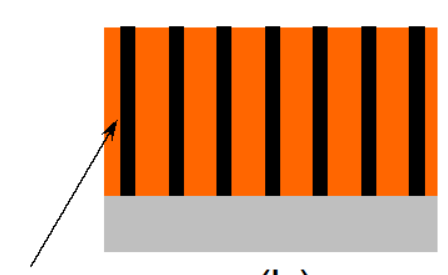

(b)

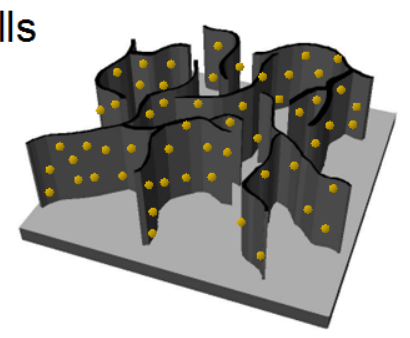

(d)

Figure 11. Schematic illustrations of decorated GNWs with different morphologies: (a) conformal deposition, (b) gap filling, (c) deposition on the top edges of GNWs, and (d) dispersed nanoparticle deposition on the surface of GNWs 
Because of the unique structure of GNWs with high surface-to-volume ratio, GNWs can be potentially used as catalyst support materials for the electrodes of fuel cells. In this application, it is required to support platinum $(\mathrm{Pt})$ nanoparticles as catalysts on the GNW surface. It is well known that the specific activity of catalysts is strongly related to their size, dispersion, and compatibility with supporting materials. Highly dispersed catalyst nanoparticles with small size and narrow size distribution supported on the surface of carbon nanostructures are ideal for high electrocatalyst activity due to their large surface-to-volume ratio. To support metal nanoparticles on the surface of carbon nanostructures, metal compounds in the form of liquids are generally employed. A few papers have been published on the preparation of Pt nanoparticles on CNT surfaces by the reduction of Pt salt precursors such as $\mathrm{H}_{2} \mathrm{PtCl}_{6}$ in solution [42,43]. However, it is difficult to treat the entire surface of GNWs with a metal compound in a liquid phase, because of the high surface tension of GNWs due to their high aspect ratio with narrow interspaces. On the other hand, in gas phase deposition such as sputtering and CVD, metal nanoparticles are deposited only around the tops of GNWs and tend to easily clump together, resulting in the formation of larger particles or films on the top of carbon nanostructures [44].

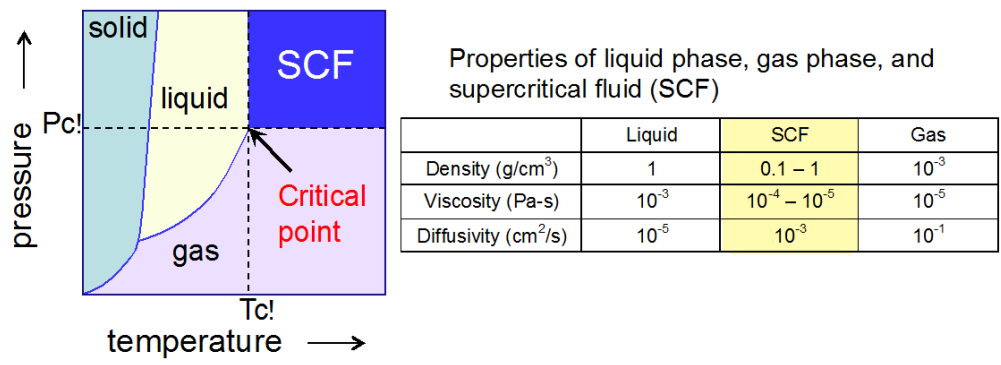

Figure 12. Phase diagram of substance (SCF supercritical fluid, Pc critical pressure, Tc critical temperature) and properties of liquid phase, gas phase, and supercritical fluid

\subsection{Supercritical fluids}

As an alternative approach to support metal nanoparticles on the surfaces of dense, aligned CNTs and GNWs with narrow interspaces, we have demonstrated a method employing metal-organic chemical fluid deposition (MOCFD), where supercritical carbon dioxide (sc$\mathrm{CO}_{2}$ ) is used as a solvent of metal-organic compounds $[45,46]$. The phase diagram of substance is shown in Fig. 12, together with the properties of liquid phase, gas phase, and supercritical fluid (SCF). Generally, materials can exist in three phases depending on the temperature and pressure, namely, solid, liquid, and gas. The SCF possesses attractive properties of both the gas and the liquid phases. Rapid diffusion and permeation are realized by its gas-like diffusivity and viscosity, while its liquid-like density enables dissolution of a wide range of materials. To produce an SCF phase, the temperature and pressure of the material are required to exceed the critical point. The critical point of sc- $\mathrm{CO}_{2}$ exists at $7.38 \mathrm{MPa}$ 
(72.8 atm) and $31.1{ }^{\circ} \mathrm{C}$. Among SCFs, $\mathrm{sc}-\mathrm{CO}_{2}$ is particularly attractive since it is environmentally friendly and safe due to its low toxicity, low reactivity and nonflammability.

In the case of Pt deposition, the SCF using sc- $\mathrm{CO}_{2}$ was first applied to the preparation of polymer-supported $\mathrm{Pt}$ nanoparticles using dimethyl (1,5-cyclooctadiene) platinum(II), $\left(\mathrm{PtMe}_{2}(\mathrm{cod})\right)$ as a precursor [47]. Erkey's group has demonstrated the preparation of $\mathrm{Pt}$ nanoparticles on a wide range of materials, including carbon aerogel, carbon black, silica aerogel, alumina, and Nafion [47-52]. In their method, $\mathrm{PtMe}_{2}(\mathrm{cod})$ was dissolved in $\mathrm{sc}-\mathrm{CO}_{2}$ and impregnated into the supporting materials, and after depressurization the impregnated $\mathrm{PtMe}_{2}(\mathrm{cod})$ molecules were then reduced to metallic Pt nanoparticles by heat treatment or by chemical reduction with hydrogen, resulting in the formation of uniformly dispersed nanoparticles with narrow size distributions. However, it took almost 10 hours to complete this process. In our case, in contrast, the supporting carbon nanostructures such as CNTs and GNWs were selectively heated in the $\mathrm{sc}-\mathrm{CO}_{2}$ with $\mathrm{Pt}$ precursors during the process. Therefore, at the heated surface of the carbon nanostructures during in situ thermal reduction under the SCF environment, decomposition of the adsorbed precursor molecules and growth of the particles would occur without reduction process.

\subsection{Experimental procedure of metal-organic chemical fluid deposition using supercritical carbon dioxide}

Figure 13 shows the SCF-MOCFD system employing $\mathrm{sc}-\mathrm{CO}_{2}$ used for the deposition of $\mathrm{Pt}$ nanoparticles on the surface of GNWs. The MOCFD system is composed of two high-pressure stainless steel vessels with a compressor, heating units, and a reservoir for the metalorganic compound. The preliminary vessel contains a screw agitator. The temperature and pressure in each vessel can be set independently, so that two different supercritical conditions employing $\mathrm{CO}_{2}$ can be produced in these two vessels. As the precursor, (methylcyclopentadienyl) trimethyl platinum $\left.\left(\left(\mathrm{CH}_{3} \mathrm{C}_{5} \mathrm{H}_{4}\right) \mathrm{Pt}\left(\mathrm{CH}_{3}\right)_{3} \text { : MeCpPtMe }\right)_{3}\right)$ dissolved in hexane was used. In the preliminary vessel, the precursor was stirred with the $\mathrm{sc}-\mathrm{CO}_{2}$ for about $30 \mathrm{~min}$. In the impregnation vessel, the selective heating of GNW samples during the MOCFD process facilitated selective metal deposition on the surface of the carbon nanostructures. In the preliminary vessel, the pressure and temperature of $\mathrm{sc}-\mathrm{CO}_{2}$ were maintained at $11 \mathrm{MPa}$ and $50^{\circ} \mathrm{C}$, respectively, and $\mathrm{MeCpPtMe}$ was dissolved in the $\mathrm{sc}-\mathrm{CO}_{2}$. In the impregnation vessel, the pressure and temperature of $\mathrm{sc}-\mathrm{CO}_{2}$ were maintained at $9 \mathrm{MPa}$ and $70^{\circ} \mathrm{C}$, respectively, and the temperature of GNW samples was controlled in the range of $70-170^{\circ} \mathrm{C}$. The solutions were mixed and Pt nanoparticles formation was carried out for $30 \mathrm{~min}$; the vessel was then depressurized slowly in $30 \mathrm{~min}$ to atmospheric conditions. After the depressurization, additional heat treatment was not carried out in the present work.

\subsection{Characterization of platinum nanoparticles formed by metal-organic chemical fluid deposition using supercritical carbon dioxide}

Figures 14(a)-14(c) show TEM images of the Pt-supported GNW surface after the SCFMOCFD at sample temperatures of 120,150 , and $170^{\circ} \mathrm{C}$, respectively. In this experiment, GNW samples were fabricated on the Si substrate by fluorocarbon $\left(\mathrm{C}_{2} \mathrm{~F}_{6}\right)$ PECVD assisted by 
hydrogen radical injection, which comprises a parallel-plate very high frequency (VHF: 100 $\mathrm{MHz}$ ), capacitively coupled plasma region, and a hydrogen radical injection source that employs a surface-wave-excited microwave $(2.45 \mathrm{GHz}) \mathrm{H}_{2}$ plasma [11]. As can be seen from these TEM images, the spatial density of the Pt nanoparticles (particle numbers/area) supported on the GNW surface strongly depended on the sample temperature during the SCFMOCFD, while the average size of the Pt nanoparticles increased from 1.5 to $3 \mathrm{~nm}$ with an increase of the sample temperature from 120 to $170^{\circ} \mathrm{C}$.

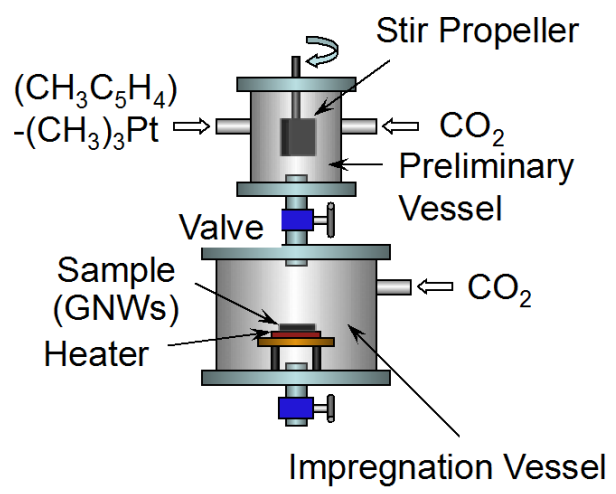

Figure 13. Schematic of the supercritical fluid, metal-organic chemical fluid deposition (SCF-MOCFD) system [53]

Ex situ X-ray photoelectron spectroscopy (XPS) analysis was conducted to gain an insight into the state of the platinum for the supported Pt surface fabricated by the SCF-MOCFD. Figure 14(d) shows an XPS spectrum of the Pt(4f) region of the Pt-supported GNW film after the SCF-MOCFD at a sample temperature of $150^{\circ} \mathrm{C}$. The presence of two prominent sets of $\mathrm{Pt}(4 f)$ peaks, corresponding to the $4 f_{7 / 2}$ and $4 f_{5 / 2}$ orbital states, is further confirmation of platinum being present on the GNW surface. The peak regions in Fig. 14(d) can be fitted with two sets of peaks at $71.4 \mathrm{eV}\left(4 f_{7 / 2}\right)$ and $74.6 \mathrm{eV}\left(4 f_{5 / 2}\right)$ [54]. These correspond to platinum in the metallic state, indicating that only pure Pt exists without being oxidized on the surface of the GNWs after the SCF-MOCFD.

The $\mathrm{Pt} / \mathrm{C}$ ratio of the GNW film surface was obtained from the ratio of the intensities of the XPS C(1s) and $\mathrm{Pt}(4 f)$ peaks. Figure 14(e) shows the relative $\mathrm{Pt} / \mathrm{C}$ ratio of the surface of the GNW film as a function of the temperature of the GNW sample during the SCF-MOCFD process. As the sample temperature during the SCF-MOCFD increased up to 120 ๑? ${ }^{\circ} \mathrm{C}$, the relative $\mathrm{Pt} / \mathrm{C}$ ratio of the surface of the GNW film increased gradually. By further increasing the sample temperature above $120^{\circ} \mathrm{C}$, the relative $\mathrm{Pt} / \mathrm{C}$ ratio increased rapidly.

\subsection{Mechanism of platinum nanoparticle formation by metal-organic chemical fluid deposition using supercritical carbon dioxide}

In the case of $\mathrm{Pt}$ deposition by the conventional process including impregnation, depressurization, and reduction, $\mathrm{PtMe}_{2}(\mathrm{cod})$ was dissolved in $\mathrm{sc}-\mathrm{CO}_{2}$ and impregnated into the sup- 
porting materials, and after depressurization the impregnated $\mathrm{PtMe}_{2}(\mathrm{cod})$ molecules were then reduced to metallic Pt nanoparticles by heat treatment or by chemical reduction with hydrogen. It was proposed that the precursor molecules in the $\mathrm{sc}-\mathrm{CO}_{2}$ phase are adsorbed on the surface during the impregnation period, and after depressurization these adsorbed molecules are in turn reduced to elemental platinum and the resulting particles at the surface continue to grow until all the adsorbed precursor molecules are converted to the metal.
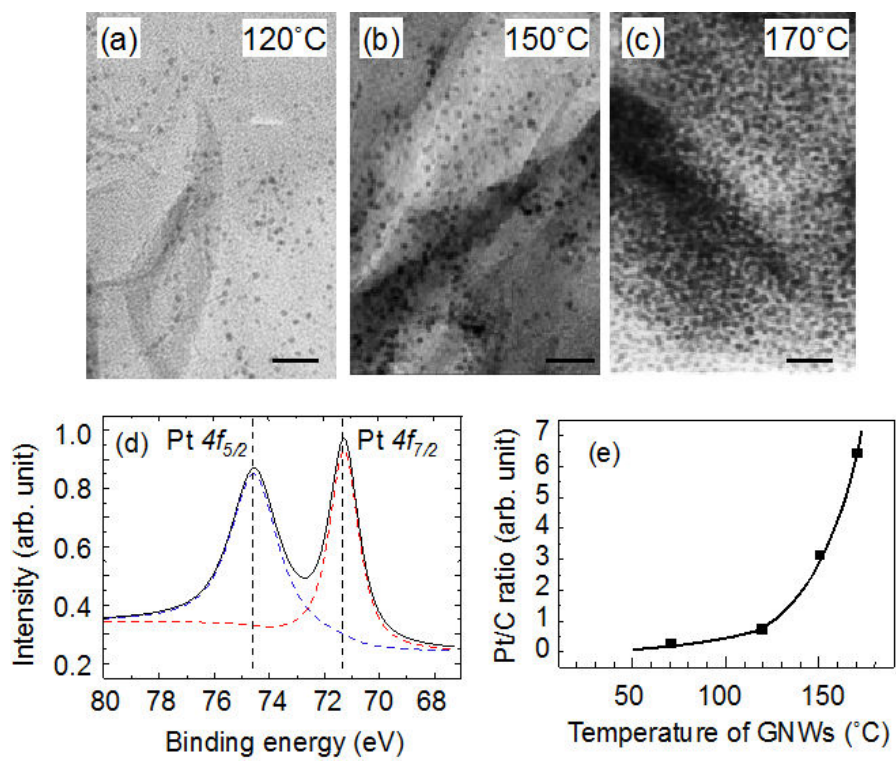

Figure 14. a)-(c) High-resolution TEM images of the surface of the GNWs supporting Pt nanoparticles after the SCFMOCFD at sample temperatures of 120,150 , and 170 월 ${ }^{\circ} \mathrm{C}$, respectively. Scale bar: $20 \mathrm{~nm}$. (d) X-ray photoelectron spectroscopy spectrum of the Pt-supported GNW film after SCF-MOCFD. (e) Relative Pt/C ratio of the surface of the GNW film as a function of temperature of the GNW sample during SCF-MOCFD [53]

In our case, in contrast, the supporting carbon nanostructures were selectively heated in the sc- $\mathrm{CO}_{2}$ with Pt precursors during the process. Figure 15 depicts the model of Pt nanoparticle formation on the surface of carbon nanostructures using metal-organic chemical fluid deposition in the supercritical $\mathrm{CO}_{2}$. At the heated surface of the carbon nanostructures during in situ thermal reduction under the SCF environment, decomposition of the adsorbed precursor molecules and growth of the particles would occur. GNWs have been reported to consist of nano-domains a few tens of nanometers in size, and individual GNWs were found to have many defects [24,55]. It is suggested that the surface-migrating $\mathrm{Pt}$ adatoms, produced by the decomposition of $\mathrm{MeCpPtMe}_{3}$ precursors, merge to form Pt clusters from several $\mathrm{Pt}$ atoms preferentially at chemically active sites such as defects and grain boundaries on the surface of the carbon nanostructures, resulting in the nucleation of Pt nanoparticles. It has been reported recently that the density of Pt nanoparticles formed by SCF-MOCFD method increased with increase in the surface defect density 
[56]. The reaction temperature at the surface would be a significant factor influencing the particle number density and particle size. When the temperature of GNWs is increased, both reduction of metal-organic precursors and surface migration of Pt atoms would be enhanced, which may lead to an increase in the particle number density and particle size. As can be seen from the TEM images in Figs. 14(a)-14(c), the average size of Pt nanoparticles increased from 1.5 to $3 \mathrm{~nm}$ with an increase in the sample temperature from 120 to $170^{\circ} \mathrm{C}$, while the $\mathrm{Pt}$ particle number density increased drastically.

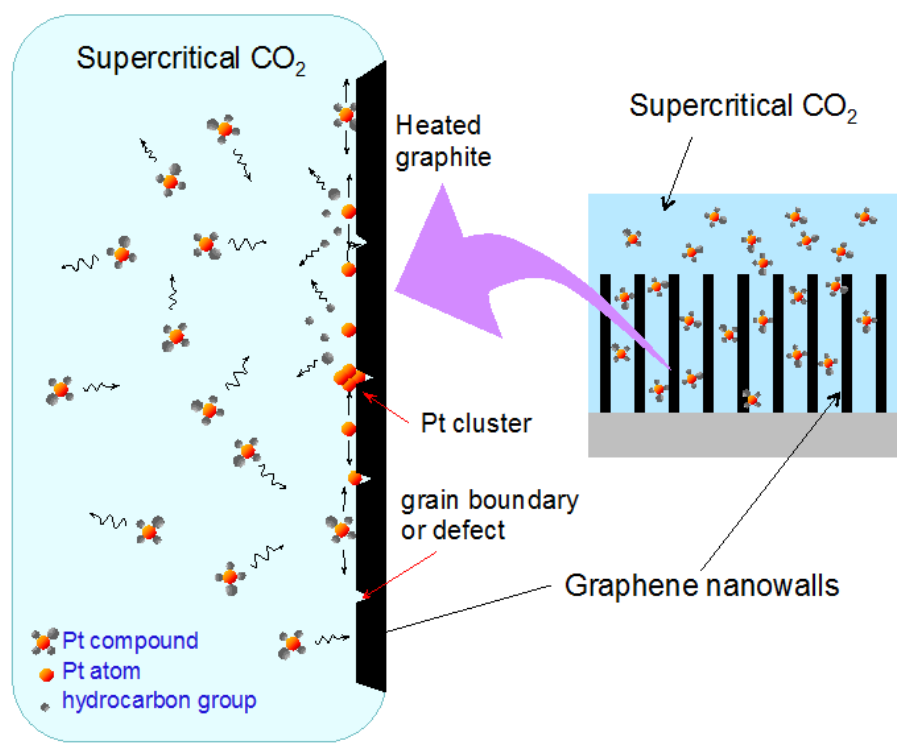

Figure 15. Illustration of the formation model of Pt nanoparticles on the surface of carbon nanostructures using metal-organic chemical fluid deposition in the supercritical $\mathrm{CO}_{2}$

\section{Fabrication of graphene nanowalls on carbon fiber paper for fuel cell application}

Carbon fiber paper (CFP) or carbon fiber cloth, which are composed of an open mesh of carbon fibers, have been used as gas diffusion layer in proton exchange membrane (PEM) fuel cell application [57-59]. PEM fuel cells have been widely recognized as the most promising candidates for future power generating devices in the automotive, distributed power generation and portable electronic applications. Waje, et al. demonstrated the preparation of $\mathrm{Pt}$ nanoparticles 2-2.5 nm in size on organically functionalized CNTs grown on CFP [60]. Very recently Lisi, et al. demonstrated the growth of GNWs on CFP by hot-filament CVD and investigated the microstructure of the GNWs both at the tip and at the fiber-nanowall base interface [61]. Our current interest in GNWs is to use them as catalyst supports for Pt in 
PEM fuel cells. In this section, in order to demonstrate the usefulness of GNWs in the fuel cell application, GNWs were directly grown on the CFP using PECVD. Subsequently, Pt nanoparticles were formed on the surface of GNWs using the SCF-MOCFD method. This configuration ensures that all the supported Pt nanoparticles are in electrical contact with the external electrical circuit. Such a design would improve Pt utilization and potentially decrease Pt usage.

\subsection{Growth of graphene nanowalls on carbon fiber paper}

Commercially available CFP (Engineered Fibers Technology, Spectracarb 2050A porous carbon-carbon paper, $200 \mu \mathrm{m}$ thick) was decorated with GNWs using RF-ICP employing $\mathrm{Ar} / \mathrm{CH}_{4}$ mixture. The growth experiments were carried out on CFP and Si substrates for 30 min at RF power of $500 \mathrm{~W}$, total gas pressure of $20 \mathrm{mTorr}$, substrate (CFP) temperature of $720{ }^{\circ} \mathrm{C}$, and flow rates of $\mathrm{Ar}$ and $\mathrm{CH}_{4}$ of 100 and $50 \mathrm{sccm}$, respectively.
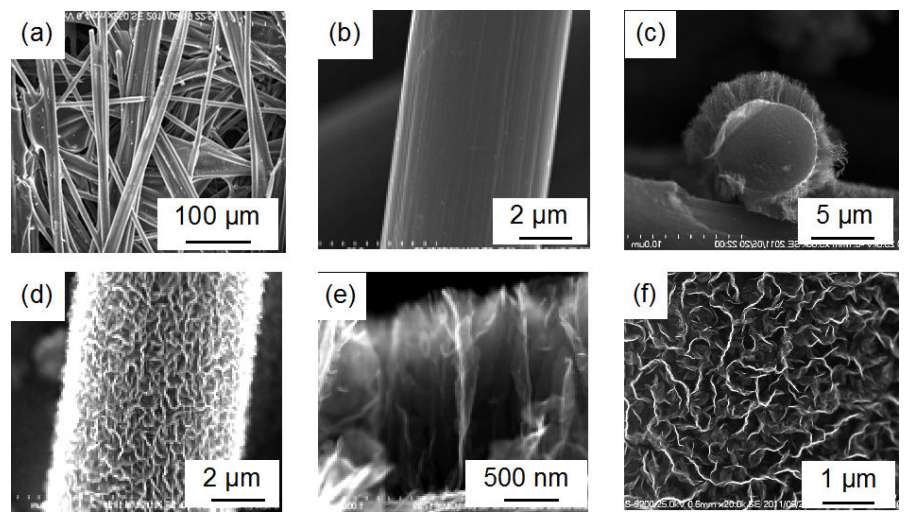

Figure 16. a), (b) SEM images of carbon fiber used in this study. (c)-(f) SEM images of GNWs grown on carbon fiber by ICP-CVD for 30 min at different magnifications [62].

Figures 16(a) and 16(b) show SEM images of carbon fiber used in this study. SEM images of GNWs grown on carbon fiber by ICP-CVD for $30 \mathrm{~min}$ at different magnifications are shown in Figs. 16(c)-16(f), indicating that GNWs were successfully grown on the CFP using ICP-CVD. As shown in Fig. 16(e), GNWs were grown almost vertically on the surface of carbon fibers forming paper structure. The height of GNWs grown on the CFP at the surface facing the plasma was about $1.5 \mu \mathrm{m}$. Interestingly the GNW growth proceeds in a conformal manner all the way around each carbon fiber into the CFP to a depth of a few tens of micrometers.

\subsection{Pt nanoparticle formation on GNW-decorated carbon fiber paper}

Pt nanoparticles were prepared on the GNWs grown on the CFP by the SCF-MOCFD method. The pressure and temperature of $\mathrm{sc}-\mathrm{CO}_{2}$ were $10 \mathrm{MPa}$ and $130{ }^{\circ} \mathrm{C}$, respectively, and the temperature of GNW-decorated CFP was maintained at $180^{\circ} \mathrm{C}$. Pt nanoparticle formation 
was carried out for $30 \mathrm{~min}$. Compared to the SEM images of typical as-grown GNW films without the SCF treatment, no change in the surface morphology was observed after the SCF treatment. It was confirmed that the unique nanostructure of the GNWs was maintained, even after being exposed to the high-pressure fluid. Figure 17(a) shows SEM image of the surface of the GNW supporting Pt nanoparticles after the SCF-MOCFD for $30 \mathrm{~min}$. It was found that dispersed Pt nanoparticles of approximately $2 \mathrm{~nm}$ in diameter were supported on the surfaces of GNWs grown on CFP. The area density of Pt nanoparticles on GNW surface was approximately $3 \times 10^{12} \mathrm{~cm}^{-2}$. Figure 17 (b) shows TEM image of the surface of the GNW supporting Pt nanoparticles after the SCF-MOCFD for $30 \mathrm{~min}$. The GNWs consist of nano-domains and individual GNWs were found to have many defects. As shown in Fig. 17(b), Pt nanoparticles were formed preferentially at the domain boundaries on the surface of the GNWs.
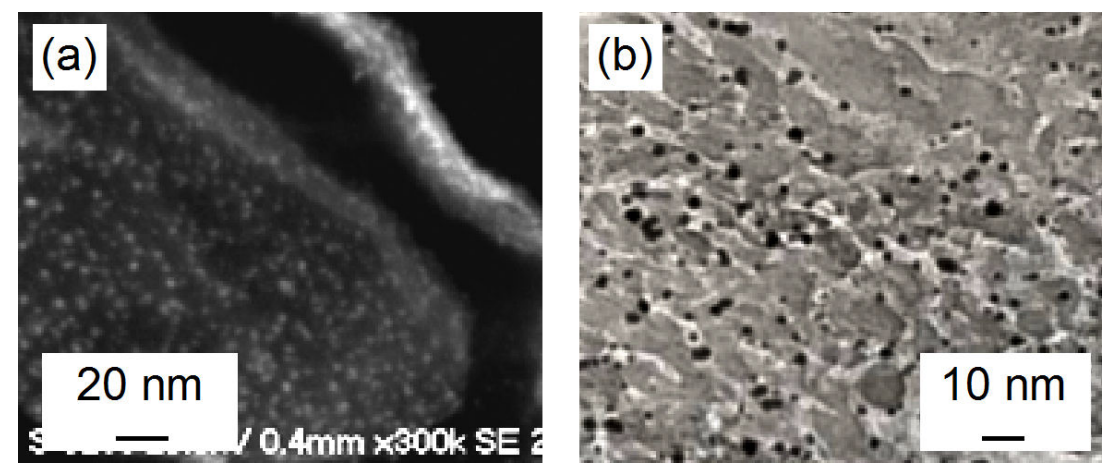

Figure 17. a) SEM image of the surface of the GNW supporting Pt nanoparticles after the SCF-MOCFD for $30 \mathrm{~min}$. (b) TEM image of the surface of the GNW supporting Pt nanoparticles after the SCF-MOCFD for 30 min

\subsection{Fuel cell unit using Pt-supported GNW/CFP electrode}

A test cell unit using Pt-supported GNW/CFP electrode was constructed experimentally. A schematic of a single PEM fuel cell with active surface area of $1 \times 1 \mathrm{~cm}^{2}$ is shown in Fig. 18 . The membrane electrode assembly (MEA) consists of a Nafion 115 membrane in combination with Pt- supported GNWs on CFPs. At present, the voltage-current curve for the test PEM fuel cell exhibited unexpectedly poor performance due to the high ohmic resistance. Very recently, Shin, et al. demonstrated the preparation of Pt nanoparticles with an average diameter of $3.5 \mathrm{~nm}$ on GNWs by a solution-reduction method, and investigated the electrocatalytic activity of powdered Pt/ GNWs peeled off from the substrate [63]. They suggested that the domain structure of GNWs is useful as catalytic support for fuel cells, although they did not demonstrate an actual PEM fuel cell using Pt/GNWs. For the evaluation of the Ptsupported GNWs on CFPs as catalyst layer for the practical use in our case, it is necessary to realize optimum operating conditions including compression, temperature, pressure, and ionomer loading. 

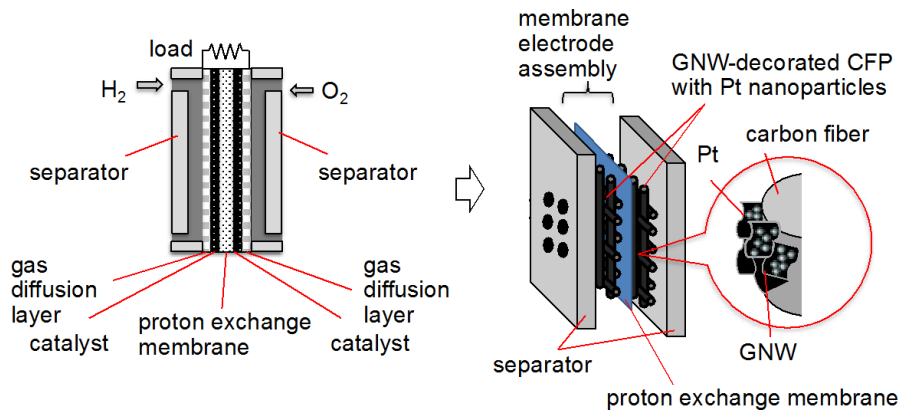

Figure 18. A schematic of a test single proton exchange membrane (PEM) fuel cell. The membrane electrode assembly (MEA) consists of a Nafion 115 membrane in combination with Pt-loaded GNWs on CFPs [62]

\section{Toward the application to electrochemical sensors and biosensors}

There has been an increase in research into the biological applications of CVD diamond. Diamond has attractive characteristics for some biological applications, such as its wide potential window, chemical-physical stability, and biocompatibility. DNA and proteins were immobilized on the surface of diamond and nanodiamond films for bio-sensing application [35-38]. Covalent modification of diamond surfaces with molecular monolayers serves as a starting point for linking biomolecules such as DNA and proteins to surfaces. In these cases, diamond surface is modified with several types of surface termination, e.g., $\mathrm{C}-\mathrm{NH}_{2}, \mathrm{C}-\mathrm{OH}$, and $\mathrm{C}-\mathrm{COOH}$. It is considered that the surfaces and edges of GNWs can also be modified with similar surface termination. Therefore, covalent immobilization of DNA and proteins on the GNWs will be realized. In the near future, GNWs will be used as a stable, highly selective platform in subsequent surface hybridization processes.

Recently, graphene has proved to be an excellent nanomaterial for applications in electrochemistry. Graphene-based materials with large surface area are useful as electrodes for electrochemical sensors and biosensors [62-64]. Especially, GNWs and related carbon nanostructures can be one of the best electrode materials to investigate basic electrochemical phenomena, due to their edge defects and graphene structures.

It is interesting to investigate the electrochemical properties of GNWs as electrochemical electrodes. In order to evaluate the potential window of GNW electrode, electrochemical measurements were carried out with a conventional three-electrode arrangement controlled by a commercial potentiostat, with a GNW (boron (B)-doped diamond for comparison) working electrode, a Pt coil counter electrode and an $\mathrm{Ag} / \mathrm{AgCl}$ reference electrode. The back face of the substrate and the electrical contact were protected from the electrolyte solution by insulating epoxy adhesive, so that only an active GNW electrode area was exposed to the electrolyte solution. Figure 19 shows the cyclic voltammograms for the GNW and B-doped diamond electrodes in $\mathrm{HNO}_{3}(0.2 \mathrm{~mol} / \mathrm{l})$ obtained in the potential range between -5 and $5 \mathrm{~V}$ 
at a scan rate of $100 \mathrm{mV} / \mathrm{s}$. As shown in Fig. 19, a work potential window nearly $3 \mathrm{~V}$ was obtained for the GNW electrode, which was comparable to that for B-doped diamond electrode. The oxygen evolution for the GNW electrodes occurs at about $1 \mathrm{~V}$ and the hydrogen evolution at $-2 \mathrm{~V}$. Reported potential windows for glassy carbon and highly oriented pyrolytic graphite electrodes are 1.5 and $2.0 \mathrm{~V}$, respectively [67].

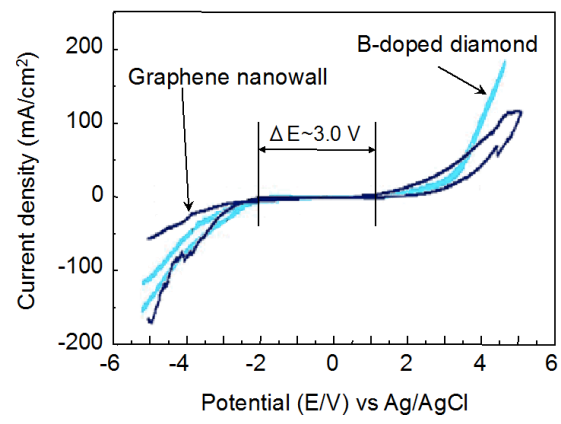

Figure 19. Cyclic voltammograms for the GNWs and boron-doped diamond electrodes in $\mathrm{HNO}_{3}(0.2 \mathrm{Mol} / \mathrm{I})$; at 100 $\mathrm{mV} / \mathrm{s}$ scan rate

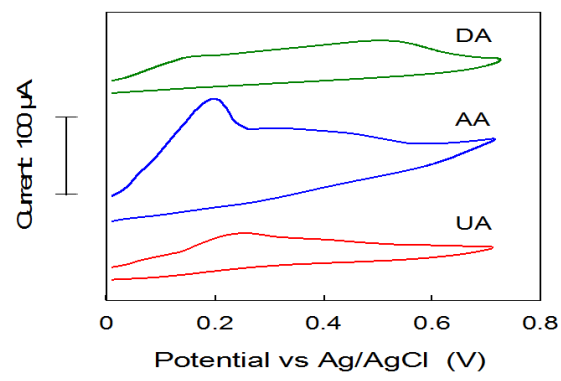

Figure 20. Cyclic voltammograms of GNW electrode in the solution of PBS with DA, AA, and UA, at $100 \mathrm{mV} / \mathrm{s} s c a n$ rate

Electrochemical activity of GNW electrode has been investigated by cyclic voltammetry measurements in an aqueous solution of ferrocyanide and a faster electron transfer between the electrolyte and the nanowall (or nanosheet) surface has been demonstrated [17-20]. Furthermore, biosensing with GNWs is a promising application. Dopamine (DA), ascorbic acid (AA), and uric acid (UA) are compounds of great biomedical interest, which all are essential biomolecules in our body fluids. Figure 20 shows cyclic voltammogram curves obtained from GNW electrode in the phosphate buffer solution (PBS) with DA, AA, and UA, at 100 $\mathrm{mV} / \mathrm{s}$ scan rate. At present, researches on the sensing of biological molecules became popular. Shang, et al. demonstrated the excellent electrocatalytic activity of multilayer graphene nanoflake films for simultaneously determining DA, AA and UA in PBS [17]. The GNWbased electrochemical platform, which possesses large surface area with edges and electro- 
chemical activity, offers great promise for providing a new class of nanostructured electrodes for biosensing and energy-conversion applications.

\section{Conclusion}

Self-organized graphite sheet nanostructures composed of graphene have been studied intensively. Graphene nanowalls and related sheet nanostructures are layered graphenes with open boundaries. The sheets form a self-supported network of wall structures with thicknesses in the range from a few nanometers to a few tens of nanometers, and with a high aspect ratio. The large surface area and sharp edges of graphene nanowalls could prove useful for a number of different applications.

Using graphene nanowalls as templates would be the most promising and important application. Graphene nanowalls can be used as templates for fabricating a variety of nanostructured materials based on the surface morphology of the graphene nanowalls and nanocomposites of carbon and nanoparticles of other materials. These structures could prove useful in batteries, sensors, solar cells, electrodes, and biomedical devices. For this purpose, it is necessary to establish decorating methods of graphene nanowall surface with a variety of materials. Furthermore, it is important to evaluate electrochemical characteristics of nanocomposites of carbon and other materials systematically.

In order to demonstrate the usefulness of graphene nanowalls in the fuel cell application, graphene nanowalls were directly grown on the carbon fiber paper using the inductively coupled plasma-enhanced chemical vapor deposition method. Subsequently, highly dispersed Pt nanoparticles $2 \mathrm{~nm}$ in size were formed on the surface of graphene nanowalls using metal-organic chemical deposition employing supercritical $\mathrm{CO}_{2}$. This configuration ensures that all the supported Pt nanoparticles are in electrical contact with the external electrical circuit. Such a design would improve Pt utilization and potentially decrease Pt usage. Pt-supported graphene nanowalls grown on the carbon fiber paper will be well suited to the application for the electrodes of fuel cells.

Furthermore, the application as a biosensor using GNWs was briefly described. The GNWbased electrochemical platform offers great promise for providing a new class of nanostructured electrodes for electrochemical sensing, biosensing and energy-conversion applications.

\section{Author details}

Mineo Hiramatsu ${ }^{1^{*}}$, Hiroki Kondo ${ }^{2}$ and Masaru Hori ${ }^{2}$

1 Meijo University, Japan

2 Nagoya University, Japan 


\section{References}

[1] Geim, A. K., \& Novoselov, K. S. (2007). The rise of graphene. Nature Materials, 6, 183-191, nmat1849.

[2] Novoselov, K. S., Geim, A. K., Morozov, S. V., Jiang, D., Zhang, Y., Dubonos, S. V., Grigorieva, I. V., \& Firsov, A. A. (2004). Electric Field Effect in Atomically Thin Carbon Films. Science, 306(5696), 666-669, science.1102896.

[3] Eda, G., Fanchini, G., \& Chhowalla, M. (2008). Large-area ultrathin films of reduced graphene oxide as a transparent and flexible electronic material. Nature Nanotechnolo$g y, 3,270-274$, nnano.2008.83.

[4] Berger, C., Song, Z. M., Li, X. B., Wu, X. S., Brown, N., Naud, C., Mayo, D., Li, T. B., Hass, J., Marchenkov, A. N., Conrad, E. H., First, P. N., \& de Heer, W. A. (2006). Electronic Confinement and Coherence in Patterned Epitaxial Graphene. Science, 312(5777), 1191-1196, science.1125925.

[5] Yu, Q., Lian, J., Siriponglert, S., Li, H., Chen, Y. P., \& Pei, S. S. (2008). Graphene segregated on Ni surfaces and transferred to insulators. Applied Physics Letters, 93(11).

[6] Li, X., Cai, W., An, J., Kim, S., Nah, J., Yang, D., Piner, R., Velamakanni, A., Jung, I., Tutuc, E., Banerjee, S. K., Colombo, L., \& Ruoff, R. S. (2009). Large-Area Synthesis of High-Quality and Uniform Graphene Films on Copper Foils. Science, 324(5932), 1312-1314.

[7] Wu, Y. H., Qiao, P. W., Chong, T. C., \& Shen, Z. X. (2002). Carbon Nanowalls Grown by Microwave Plasma Enhanced Chemical Vapor Deposition. Advanced Materials, 14(1), 64-67, AID- ADMA64>3.0.CO;2-G.

[8] Hiramatsu, M., Shiji, K., Amano, H., \& Hori, M. (2004). Fabrication of vertically aligned carbon nanowalls using capacitively coupled plasma-enhanced chemical vapor deposition assisted by hydrogen radical injection. Applied Physics Letters, 84(23), $4708-4710$.

[9] Wang, J. J., Zhu, M. Y., Outlaw, R. A., Zhao, X., Manos, D. M., Holloway, B. C., \& Mammana, V. P. (2004). Free-standing subnanometer graphite sheets. Applied Physics Letters, 85(7), 1265, 10.1063/1.1782253.

[10] Hiramatsu, M., \& Hori, M. (2006). Fabrication of Carbon Nanowalls Using Novel Plasma Processing. Japanese Journal of Applied Physics, 45, 5522-5527, JJAP.45.5522.

[11] Kondo, S., Hori, M., Yamakawa, K., Den, S., Kano, H., \& Hiramatsu, M. (2008). Highly reliable growth process of carbon nanowalls using radical injection plasma-enhanced chemical vapor deposition. Journal of Vacuum Science \& Technology B, 26(4), 1294-1300. 
[12] Mori, T., Hiramatsu, M., Yamakawa, K., Takeda, K., \& Hori, M. (2008). Fabrication of carbon nanowalls using electron beam excited plasma-enhanced chemical vapor deposition. Diamond and Related Materials, 17(7-10), 1513-1517, j.diamond.2008.01.070.

[13] Chuang, A. T. H., Robertson, J., Boskovic, B. O., \& Koziol, K. K. K. (2007). Three-dimensional carbon nanowall structures. Applied Physics Letters, 90(12), 123107.

[14] Hou, K., Outlaw, R. A., Wang, S., Zhu, M., Quinlan, R. A., Manos, D. M., Kordesch, M. E., Arp, U., \& Holloway, B. C. (2008). Uniform and enhanced field emission from chromium oxide coated carbon nanosheets. Applied Physics Letters, 92(13).

[15] Yang, B. J., Wu, Y. H., Zong, B. Y., \& Shen, Z. X. (2002). Electrochemical Synthesis and Characterization of Magnetic Nanoparticles on Carbon Nanowall Templates. Nano Letters, 2(7), 751-754, nl025572r.

[16] Giorgi, L., Makris, T. D., Giorgi, R., Lisi, N., \& Salernitano, E. (2007). Electrochemical properties of carbon nanowalls synthesized by HF-CVD. Sensors and Actuators B: Chemical, 126(1), 144-152, j.snb.2006.11.018.

[17] Shang, N. G., Papakonstantinou, P., Mc Mullan, M., Chu, M., Stamboulis, A., Potenza, A., Dhesi, S. S., \& Marchetto, H. (2008). Catalyst-Free Efficient Growth, Orientation and Biosensing Properties of Multilayer Graphene Nanoflake Films with Sharp Edge Planes. Advanced Functional Materials, 18(21), 3506-3514, adfm.200800951.

[18] Luais, E., Boujtia, M., Gohier, A., Tailleur, A., Casimirius, S., Djouadi, M. A., Granier, A., \& Tessier, P. Y. (2009). Carbon nanowalls as material for electrochemical transducers. Applied Physics Letters, 95(1), 014104.

[19] Tanaike, O., Kitada, N., Yoshimura, H., Hatori, H., Kojima, K., \& Tachibana, M. (2009). Lithium insertion behavior of carbon nanowalls by dc plasma CVD and its heat-treatment effect. Solid State Ionics, 180(4-5), 381-385, j.ssi.2009.01.012.

[20] Wang, Z., Shoji, M., \& Ogata, H. (2011). Carbon nanosheets by microwave plasma enhanced chemical vapor deposition in CH4-Ar system. Applied Surface Science, 257(21), 9082-9085, j.apsusc.2011.05.104.

[21] Hiramatsu, M., Nihashi, Y., Kondo, H., \& Hori, M. (2012). Nucleation control of selforganized vertical nano-graphenes using inductively coupled plasma enhanced chemical vapor deposition. Japanese Journal of Applied Physics, 51 (to be published), 51.

[22] Nemanich, R. J., \& Solin, S. A. (1979). First- and second-order Raman scattering from finite-size crystals of graphite. Physical Review B, 20(2), 392-401, PhysRevB.20.392.

[23] Kurita, S., Yoshimura, A., Kawamoto, H., Uchida, T., Kojima, K., Tachibana, M., Molina-Morales, P., \& Nakai, H. (2005). Raman spectra of carbon nanowalls grown by plasma-enhanced chemical vapor deposition. Journal of Applied Physics, 97(10).

[24] Ferrari, A. C., Meyer, J. C., Scardaci, V., Casiraghi, C., Lazzeri, M., Mauri, F., Piscanec, S., Jiang, D., Novoselov, K. S., Roth, S., \& Geim, A. K. (2006). Raman Spectrum of Graphene and Graphene Layers. Physical Review Letters, 97, PhysRevLett.97.187401. 
[25] Ferrari, A. C. (2007). Raman spectroscopy of graphene and graphite: Disorder, electron-phonon coupling, doping and nonadiabatic effects. Solid State Communications, 143(1-2), 47-57, j.ssc.2007.03.052.

[26] Kawata, M., Nadahara, S., Shinozawa, J., Watanabe, M., \& Katoda, T. (1990). Characterization of stress in doped and undoped polycrystalline silicon before and after annealing or oxidation with laser raman spectroscopy. Journal of Electronic Materials, 19(5), 407-411, BF02657998.

[27] Kondo, S., Kawai, S., Takeuchi, W., Yamakawa, K., Den, S., Kano, H., Hiramatsu, M., \& Hori, M. (2009). Initial growth process of carbon nanowalls synthesized by radical injection plasma-enhanced chemical vapor deposition. Journal of Applied Physics, 106(9), 10.1063/1.3253734.

[28] Kawai, S., Kondo, S., Takeuchi, W., Kondo, H., Hiramatsu, M., \& Hori, M. (2010). Optical Properties of Evolutionary Grown Layers of Carbon Nanowalls Analyzed by Spectroscopic Ellipsometry. Japanese Journal of Applied Physics, 49, JJAP.49.060220.

[29] Kondo, S., Kondo, H., Hiramatsu, M., Sekine, M., \& Hori, M. (2010). Critical Factors for Nucleation and Vertical Growth of Two Dimensional Nano-Graphene Sheets Employing a Novel Ar+ Beam with Hydrogen and Fluorocarbon Radical Injection. Applied Physics Express, 3, APEX.3.045102.

[30] Zhao, X., Outlaw, R. A., Wang, J. J., Zhu, M. Y., Smith, G. D., \& Holloway, B. C. (2006). Thermal desorption of hydrogen from carbon nanosheets. Journal of Chemical Physics, 124(19).

[31] Zhu, M., Wang, J., Holloway, B. C., Outlaw, R. A., Zhao, X., Hou, K., Shutthanandan, V., \& Manos, D. M. (2007). A mechanism for carbon nanosheet formation. Carbon, 45(11), 2229-2234, j.carbon.2007.06.017.

[32] Seo, D. H., Kumar, S., \& Ostrikov, K. (2011). Control of morphology and electrical properties of self-organized graphenes in a plasma. Carbon, 49(13), 4331-4339, j.carbon.2011.06.004.

[33] Krivchenko, V. A., Dvorkin, V. V., Dzbanovsky, N. N., Timofeyev, M. A., Stepanov, A. S., Rakhimov, A. T., Suetin, N. V., Vilkov, O. Y., \& Yashina, L. V. (2012). Evolution of carbon film structure during its catalyst-free growth in the plasma of direct current glow discharge. Carbon, 50(4), 1477-1487, j.carbon.2011.11.018.

[34] Ushizawa, K., Sato, Y., Mitsumori, T., Machinami, T., Ueda, T., \& Ando, T. (2002). Covalent immobilization of DNA on diamond and its verification by diffuse reflectance infrared spectroscopy. Chemical Physics Letters, 351(1-2), 105-108, S0009-2614(01)01362-8.

[35] Yang, W., Auciello, O., Butler, J. E., Cai, W., Carlisle, J. A., Gerbi, J. E., Gruen, D. M., Knickerbocker, T., Lasseter, T. L., Russell, J. N., Smith, L. M., \& Hamers, R. J. (2002). DNA-modified nanocrystalline diamond thin-films as stable, biologically active substrates. Nature Materials, 1(4), 253-257, nmat779. 
[36] Wenmackers, S., Haenen, K., Nesladek, M., Wagner, P., Michiels, L., van de Ven, M, \& Ameloot, M. (2003). Covalent immobilization of DNA on CVD diamond films. Physica Status Solidi (a), 199(1), 44-48, pssa.200303822.

[37] Takahashi, K., Tanga, M., Takai, O., \& Okamura, H. (2003). DNA preservation using diamond chips. Diamond and Related Materials, 12(3-7), 572-576, S0925-9635(03)00070-0.

[38] Wu, Y. H., Yang, B. J., Zong, B. Y., Sun, H., Shen, Z. X., \& Feng, Y. P. (2004). Carbon nanowalls and related materials. Journal of Materials Chemistry, 14(4), 469-477, B311682D.

[39] Yang, B. J., Wu, Y. H., Zong, B. Y., \& Shen, Z. X. (2002). Electrochemical synthesis and characterization of magnetic nanoparticles on carbon nanowall templates. Nano Letters, 2(7), 751-754, nl025572r.

[40] Wu, Y. H., Yang, B. J., Han, G. C., Zong, B. Y., Ni, H. Q., Luo, P., Chong, T. C., Low, T. S., \& Shen, Z. X. (2002). Fabrication of a class of nanostructured materials using carbon nanowalls as the templates. Advanced Functional Materials, 12(8), 489-494, AIDADFM489>3.0.CO;2-X.

[41] Huang, J. E., Guo, D. J., Yao, Y. G., \& Li, H. L. (2005). High dispersion and electrocatalytic properties of platinum nanoparticles on surface-oxidized single- walled carbon nanotubes. Journal of Electroanalytical Chemistry, 577(1), 93-97, j.jelechem. 2004.11.019.

[42] Mu, Y., Liang, H., Hu, J., Jiang, L., \& Wan, L. (2005). Controllable Pt Nanoparticle Deposition on Carbon Nanotubes as an Anode Catalyst for Direct Methanol Fuel Cells. Journal of Physical Chemistry B, 109(47), 22212-22216, jp0555448.

[43] Rabat, H., Andreazza, C., Brault, P., Caillard, A., Béguin, F., Charles, C., \& Boswell, R. (2009). Carbon/platinum nanotextured films produced by plasma sputtering. Carbon, 47(1), 209-214, j.carbon.2008.09.051.

[44] Machino, T., Takeuchi, W., Kano, H., Hiramatsu, M., \& Hori, M. (2009). Synthesis of platinum nanoparticles on two-dimensional carbon nanostructures with an ultrahigh aspect ratio employing supercritical fluid chemical vapor deposition process. Applied Physics Express, 2, APEX.2.025001.

[45] Hiramatsu, M., \& Hori, M. (2010). Preparation of Dispersed Platinum Nanoparticles on a Carbon Nanostructured Surface Using Supercritical Fluid Chemical Deposition. Materials, 3(3), 1559-1572, ma3031559.

[46] Watkins, J. J., \& Mc Carthy, T. J. (1995). Polymer/Metal Nanocomposite Synthesis in Supercritical $\mathrm{CO}_{2}$. Chemistry of Materials, 7(11), 1991-1994, cm00059a001.

[47] Saquing, C. D., Kang, D., Aindow, M., \& Erkey, C. (2005). Investigation of the supercritical deposition of platinum nanoparticles into carbon aerogels. Microporous and Mesoporous Materials, 80(1-3), 11-23, j.micromeso.2004.11.019. 
[48] Zhang, Y., Kang, D., Saquing, C. D., Aindow, M., \& Erkey, C. (2005). Supported Platinum Nanoparticles by Supercritical Deposition. Industrial \& Engineering Chemistry Research, 44(11), 4161-4164, ie050345w.

[49] Zhang, Y., \& Erkey, C. (2006). Preparation of supported metallic nanoparticles using supercritical fluids: A review. The Journal of Supercritical Fluids, 38(2), 252-267, j.supflu.2006.03.021.

[50] Bayrakceken, A., Kitkamthorn, U., Aindow, M., \& Erkey, C. (2007). Decoration of multi-wall carbon nanotubes with platinum nanoparticles using supercritical deposition with thermodynamic control of metal loading. Scripta Materialia, 56(2), 101-103, 10.1016/j.scriptamat.2006.09.019.

[51] Erkey, C. (2009). Preparation of metallic supported nanoparticles and films using supercritical fluid deposition. Journal of Supercritical Fluids, 47(3), 517-522, j.supflu. 2008.10.019.

[52] Hiramatsu, M., Machino, T., Mase, K., Hori, M., \& Kano, H. (2010). Preparation of Platinum Nanoparticles on Carbon Nanostructures Using Metal-Organic Chemical Fluid Deposition Employing Supercritical Carbon Dioxide. Journal of Nanoscience and Nanotechnology, 10(6), 4023-4029, jnn.2010.1996.

[53] Pitchon, V., \& Fritz, A. (1999). The relation between surface state and reactivity in the $\mathrm{DeNO}_{\mathrm{x}}$ mechanism on platinum-based catalysts. Journal of catalysis, 186(1), 64-74, jcat. 1999.2543.

[54] Kobayashi, K., Tanimura, M., Nakai, H., Yoshimura, A., Yoshimura, H., Kojima, K., \& Tachibana, M. (2007). Nanographite domains in carbon nanowalls. Journal of Applied Physics, 101(9).

[55] Mase, K., Kondo, H., Kondo, S., Hori, M., Hiramatsu, M., \& Kano, H. (2011). Formation and mechanism of ultrahigh density platinum nanoparticles on vertically grown graphene sheets by metal-organic chemical supercritical fluid deposition. Applied Physics Letters, 98(19).

[56] Zhang, X., \& Shen, Z. (2002). Carbon fiber paper for fuel cell electrode. Fuel, 81(17), 2199-2201, S0016-2361(02)00166-7.

[57] Wang, L., Husar, A., Zhou, T., \& Liu, H. (2003, International Journal of Hydrogen Energy). A parametric study of PEM fuel cell performances. 28(11), 1263-1272, DOI:S0360-3199(02)00284-7.

[58] Ge, J., Higier, A., \& Liu, H. (2006). Effect of gas diffusion layer compression on PEM fuel cell performance. Journal of Power Sources, 159(2), 922-927, j.jpowsour.2005.11.069.

[59] Waje, M. M., Wang, X., Li, W., \& Yan, Y. (2005). Deposition of platinum nanoparticles on organic functionalized carbon nanotubes grown in situ on carbon paper for fuel cells. Nanotechnology, 16, S395-S400.

[60] Lisi, N., Giorgi, R., Re, M., Dikonimos, T., Giorgi, L., Salernitano, E., Gagliardi, S., \& Tatti, F. (2011). Carbon nanowall growth on carbon paper by hot filament chemical 
vapour deposition and its microstructure. Carbon, 49(6), 2134-2140, j.carbon. 2011.01.056.

[61] Shin, S. C., Yoshimura, A., Matsuo, T., Mori, M., Tanimura, M., Ishihara, A., Ota, K., \& Tachibana, M. (2011). Carbon nanowalls as platinum support for fuel cells. Journal of Applied Physics, 110(10).

[62] Zhou, M., Zhai, , \& Dong, S. (2009). Electrochemical Sensing and Biosensing Platform Based on Chemically Reduced Graphene Oxide. Analytical Chemistry, 81(14), 5603-5613, ac900136z.

[63] Pumera, M., Ambrosi, A., Bonanni, A., Chng, E. L. K., \& Poh, H. L. (2010). Graphene for electrochemical sensing and biosensing. Trends in Analytical Chemistry, 29(9), 954-965, j.trac.2010.05.011.

[64] Hill, E. W. (2011). Graphene Sensors. IEEE Sensors Journal, 11(12), 3161-3170, JSEN. 2011.2167608.

[65] Fujishima, A., Einaga, Y., Rao, T. N., \& Tryn, D. A. (2004). Diamond electrochemistry, BKC Inc./ Elsevier BV, Tokyo/Amsterdam, 28. 